\title{
Toward short-lived and energy-dependent fission product yields from neutron-induced fission
}

\author{
Anton P. Tonchev ${ }^{1,4, *}$, Jack A. Silano ${ }^{1}$, Chris Hagmann ${ }^{1}$, Roger Henderson ${ }^{1}$, Mark A. Stoyer ${ }^{1}$, Matthew Gooden ${ }^{2}$, \\ Todd Bredeweg ${ }^{2}$, Jerry Wilhelmy ${ }^{2}$, Werner Tornow ${ }^{3,4}$, Calwin Howell ${ }^{3,4}$, Sean Finch ${ }^{3,4}$, and FNU Krishichayan ${ }^{3,4}$ \\ ${ }^{1}$ Lawrence Livermore National Laboratory, Livermore, California 94550, USA \\ ${ }^{2}$ Los Alamos National Laboratory, P.O. Box 1663, Los Alamos, NM 87545, USA \\ ${ }^{3}$ Triangle Universities Nuclear Laboratory, Durham, North Carolina, 27708, USA \\ ${ }^{4}$ Department of Physics, Duke University, Durham North Carolina, 27708, USA
}

\begin{abstract}
Fission product yields (FPYs) are an important source of information that are used for basic and applied physics. They are essential observables to address questions relevant to nucleosynthesis in the cosmos that created the elements from iron to uranium, for example, in energy generating processes from fission recycling in binary neutron star mergers; resolving the reactor neutrino anomaly; decay heat release in nuclear reactors; and many national security applications. While new applications will require accurate energy-dependent FPY data over a broad set of incident neutron energies, the current evaluated FPY data files contain only three energy points: thermal, fast, and 14-MeV incident energies.

Recent measurements using mono-energetic and pulsed neutron beams at the Triangle Universities Nuclear Laboratory (TUNL) tandem accelerator and employing a dual fission ionization chambers setup have produced self-consistent, high-precision data critical for testing fission models for the neutron-induced fission of the major actinide nuclei. This paper will present new campaign just beginning utilizing a RApid Belt-driven Irradiated Target Transfer System (RABITTS) to measure shorter-lived fission products and the time dependence of fission yields, expanding the measurements from cumulative towards independent fission yields.
\end{abstract}

\section{Introduction}

Nuclear fission is a collective phenomenon in which a heavy parent nucleus splits into two daughter nuclei either spontaneously or as a result of induced fission. The distribution of fragment masses following fission is one of the most basic quantities that has been observed since the discovery of fission by Hahn and Strassmann in 1938, and these yields play an important role in many applications such as the estimation of decay heat and delayed neutron emission in nuclear reactors, reactor neutrino studies, radio isotope production for medical applications, development of advanced reactor and transmutation systems, fission in the galactic chemical evolution, national security, among others. For these reasons, a demand for highquality fission product yield (FPY) data in such applications is rapidly increasing. Unfortunately, due to the complexity of the fission phenomenon, the mechanism of mass distribution is not simple and has been a challenge for fission theory using microscopic or phenomenological concepts [1]. While new applications will require accurate energy-dependent FPY data over a broad range of incident neutron energies, the current evaluated FPY data files contain only three energy points: thermal, fast $(\sim 2 \mathrm{MeV})$, and $14-\mathrm{MeV}$ incident energies. Currently there is a renewed

*e-mail: tonchev2@1lnl.gov effort from the fission community to extend and improve this FPY data.

\section{Fission product-yield measurements}

Neutron-induced fission, such as the ${ }^{235} \mathrm{U}(\mathrm{n}, \mathrm{f})$ or ${ }^{239} \mathrm{Pu}(\mathrm{n}, \mathrm{f})$ reactions, produces roughly 800 primary fission fragments, for which both the experimental data and the modeling represent a critical part of the FPY data evaluation [2]. Due to the intrinsic complexity of the fission mechanism, the dependence of fission yields on incident neutron energy is a very difficult problem. At present there are still many open questions and further studies in experiment and theory are needed to make quantitative predictions for the FPYs [3, 4].

It is useful to define several terms related to fission product yields. The term "fission fragments" is used before the emission of prompt neutrons. On the contrary the term "fission products" refer to the nuclear species after emission of prompt neutrons and prompt $\gamma$-rays. The fission products decay by emitting $\beta$-particles, $\gamma$-rays and delayed neutrons. The independent product yield, $Y_{i}(A, Z)$, of a nuclide is the number of atoms with this given $A$ and $Z$ produced directly from fission before any radioactive decay. The cumulative yield, $Y_{c}(A, Z)$, specifies the total number of atoms of nuclide $(A, Z)$ produced in a specific fission by directly summing its independent yield with the 
independent yields of all its precursors. Obviously $Y_{i}$ and $Y_{c}$ are interconnected by the decay data, such as the $\beta$ decay branching ratios, delayed neutron emission probabilities, isomeric state production ratios, etc. The total chain yield, $Y_{c h}(A, Z)$, is equal to the cumulative yield of the last member of the isobaric chain. The fission yields are typically measured by one of three methods: (1) direct high-resolution $\gamma$-ray spectroscopy, (2) radiochemical separation of the fission products followed by $\beta$ and $\gamma$-ray counting, and (3) mass separation using of the socalled inverse kinematics of stopped and unstopped techniques. The last technique is used more for extracting the independent FPYs however, even the accurate determination of the compound nucleus composition is rather difficult due to the complexity of the excitation process of the nuclide [5]. Hence, direct measurements of independent yields are very limited in energy, fissioning system and resolution of the fragment mass and charge distributions. For this paper, we focus on the first method where fractional-independent or -cumulative fission yields are generally obtained by the analysis of growth and decay curves of daughters and parents in $\gamma$-ray spectroscopy. In the past, systematic studies by Livermore [6-9], Argonne [10-12], and Oak Ridge [13-16] groups provided FPY information for a large number of fissioning systems. While there are a number of energy-integral measurements of cumulative fission product yields (e.g., following $\beta$ decay of the fission products themselves), particularly for thermal neutrons $[14,17,18]$, data for higher-energy $\left(E_{n}>1\right.$ $\mathrm{MeV}$ ) differential measurements of short-lived FPYs are incomplete or have substantial uncertainties due to the low-intensity of quasi-monoenergetic fast neutron sources and the short lifetimes of fission products far from the valley of stability.

\subsection{From cumulative to short-lived FPY}

We aim to extend our experimental capability of measuring not just the total chain yield $Y_{c h}(A, Z)$, i.e. the last or the longest-lived radioactive member of the $\beta$-decay chain, but the energy dependency of the cumulative $Y_{c}(A, Z)$ and independent fission yields $Y_{I}(A, Z)$ using monoenergetic neutron beams coupled with high-resolution $\gamma$-ray spectroscopy. This can be achieved by applying different short irradiation and counting times to optimize FPY information for 5-6 precursors removed from the line of stability. As an example, the fission chain for mass number $A=133$ is shown in Fig. 1 in order to illustrate the range of fission product isotopes accessible by this technique. Measurements of these short-lived FPYs require new experimental techniques, such as developing and commissioning a new fast transport system at TUNL to produce complete and consistent yields for fissioning systems important for basic fission theory and applications. These high-precision and energy-dependent FPY data can bridge our measured cumulative long-lived chain fission yields with the independent FPYs by using a time-dependent method, e.g. the Bateman equations, describing activities in a decay chain as a function of time, based on the decay rates and initial abundances [19].

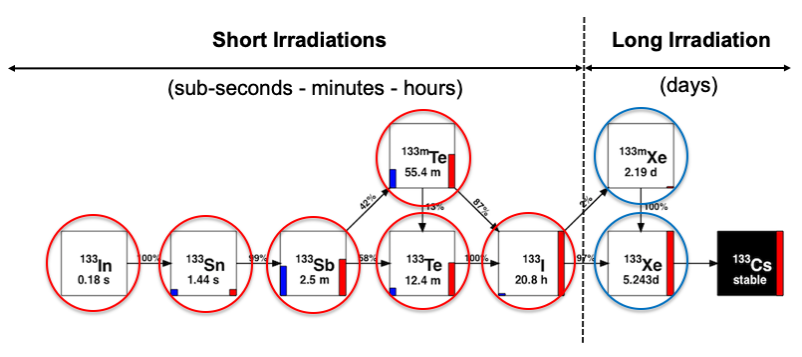

Figure 1. Fission chain yields $Y_{c h}(A)$ (circled in blue) presently measured at TUNL using long ( $\sim$ one week) irradiation. Shortand very-short-lived fission yields $\mathrm{Y}_{c}(A, Z)$ (circled in red) - to be measured at TUNL using the proposed fast-transport "RABITT" system. The vertical blue and red bars illustrate the evolution of the corresponding independent and cumulative FPYs along the isobaric mass chain for $A=133$ [20].

\subsection{Energy dependence of cumulative (chain) fission yields}

Since 2011 the LLNL-LANL-TUNL collaboration has undertaken experimental measurements of the energy evolution of the chain fission-product yields from neutroninduced fission of ${ }^{235} \mathrm{U},{ }^{238} \mathrm{U}$, and ${ }^{239} \mathrm{Pu}$ using quasimonoenergetic neutrons produced at the TUNL $10 \mathrm{MV}$ Van de Graaff accelerator. Our method relies on direct (instrumental), post-irradiation $\gamma$-ray spectroscopy of $\gamma$ rays emitted by fission products in actinide targets, with multiple $\gamma$-ray spectra taken for a period of at least a few days. High yields of fast monoenergetic neutrons between 0.2 and $15 \mathrm{MeV}$ are produced utilizing the four nuclear reactions ${ }^{7} \mathrm{Li}(\mathrm{p}, \mathrm{n}){ }^{7} \mathrm{Be},{ }^{3} \mathrm{H}(\mathrm{p}, \mathrm{n}){ }^{3} \mathrm{He},{ }^{2} \mathrm{H}(\mathrm{d}, \mathrm{n})^{3} \mathrm{He}$, and ${ }^{3} \mathrm{H}(\mathrm{d}, \mathrm{n})^{4} \mathrm{He}$. A schematic of the experimental setup is shown in Fig. 2. During exposure to the neutron beam, dual fission chambers (DFC) with the same isotope as the target of interest have been used to monitor the total fission rate [21]. The chambers contain two thin $\left(\sim 100 \mu \mathrm{g} / \mathrm{cm}^{2}\right)$ reference foils and a thicker $\left(\sim 200 \mathrm{mg} / \mathrm{cm}^{2}\right)$ actinide activation target [22]. The activation target is contained in the center of the chamber while the thin reference foils are up- and down-stream from the activation target. The thick activation target is composed of the same actinide material as the thin reference foils in the adjacent chambers. The advantage of using the DFC method, compared to other methods such as radiochemistry, ratio methods, or mass separation, is that the fission chamber determines the total number of fissions in the target without having to explicitly know either the fission cross section or the neutron flux, greatly reducing the total uncertainty of the measurements. At the same time, performing high-resolution $\gamma$-ray counting of the actinide activation target provides the ultimate isotope resolution in $(A)$ and $(Z)$. Thus the ratio of the individual fission-fragment $\gamma$-ray yields and the total number of fissions, measured by the DFC, gives the FPYs.

The main experimental results of this study have been published in Refs. [23-25]. FPY measurements have been made on the three isotopes of interest at eight incident neutron energies: $0.58,1.37,2.37,3.6,4.6,5.5,8.9$ and 14.8 $\mathrm{MeV}$. Recently, these FPY measurements were aslo com- 


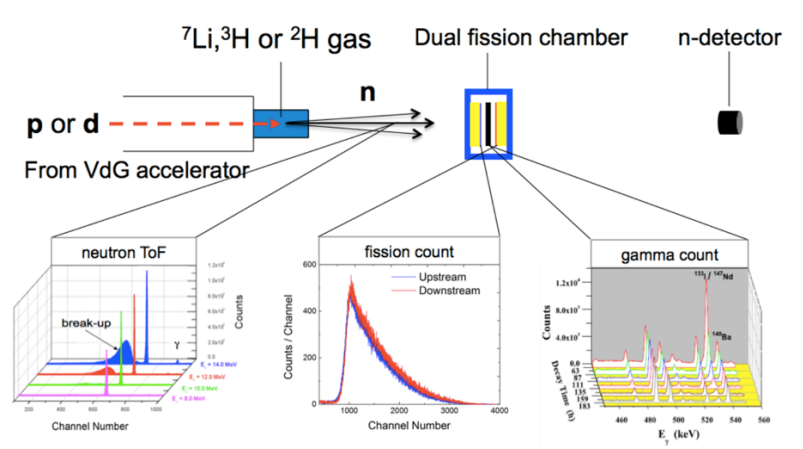

Figure 2. Schematic of the FPY experimental setup at TUNL. The bottom left panel shows the TOF neutron spectra measured, the bottom middle panel, the DFC pulse-height spectrum, and the right panel shows the time evolution of the $\gamma$-ray spectra.

pleted at $E_{n}=6.5,7.5,11.0$, and $13.0 \mathrm{MeV}$ [24]. Typically, irradiation times ranged from 4-6 days depending on neutron flux and fission cross section, followed by continuous $\gamma$-ray counting of fission product induced activity. For each incident energy, approximately 16 cumulative chain FPYs were determined, nearly all high-yield fission products, i.e., those occurring in the peaks of the mass distributions. Our FPY results show that cumulative chain yields of long-lived fission products are strongly dependent on the target nucleus and the neutron energy. The high-accuracy (2-4\%) of the FPY measurements, over the energy range of 0.58 to $14.8 \mathrm{MeV}$, reveals an anomalous energy dependence in some FPYs. As an example, the experimental results from six high-yield fission products ${ }^{95,97} \mathrm{Zr},{ }^{99} \mathrm{Mo},{ }^{140} \mathrm{Ba},{ }^{143} \mathrm{Ce}$, and ${ }^{147} \mathrm{Nd}$ from neutron induced fission of ${ }^{239} \mathrm{Pu},{ }^{235} \mathrm{U}$, and ${ }^{238} \mathrm{U}$ are presented in Fig. 3. As can been seen, the FPY of ${ }^{147} \mathrm{Nd}$ (bottom right panel) shows a positive energy trend for $E_{n}<5 \mathrm{MeV}$. This trend is almost linear up to about $4 \mathrm{MeV}$, confirming the earlier assessment from critical assembly measurements for fission neutrons below $E_{n}=2 \mathrm{MeV}$ [26, 27]. At higher energies the slope of the FPY for ${ }^{147} \mathrm{Nd}$ turns over and becomes negative. An unexpected observation is that a small, positive slope is observed for most other high-yield fission products that were studied from 0.58 to $3.6 \mathrm{MeV}$ in ${ }^{239} \mathrm{Pu}(\mathrm{n}, \mathrm{f})$ and ${ }^{238} \mathrm{U}(\mathrm{n}, \mathrm{f})$. The strongest overall energy dependence was seen in neutron induced fission of ${ }^{239} \mathrm{Pu}$. However, as mentioned above, similar positive slopes were obtained from the study of ${ }^{238} \mathrm{U}$ as well. In the case of ${ }^{235} \mathrm{U}(\mathrm{n}, \mathrm{f})$ the energy dependence of the FPYs show constant or slightly negative slope from 0.5 to $5 \mathrm{MeV}$. The only common feature of all three target isotopes is the decrease of the FPYs with higher incident neutron energy $E_{n}>5 \mathrm{MeV}$, attributed to an increased symmetric fission component. Currently, there are ongoing theoretical efforts based on nuclear density functional theory or other comprehensive microscopic frameworks to shed light on this intriguing energy dependence [28, 29].

Our previous experiments have significantly improved the precision of the determination of the energy dependence of the fission chain yields. However, since our ir-
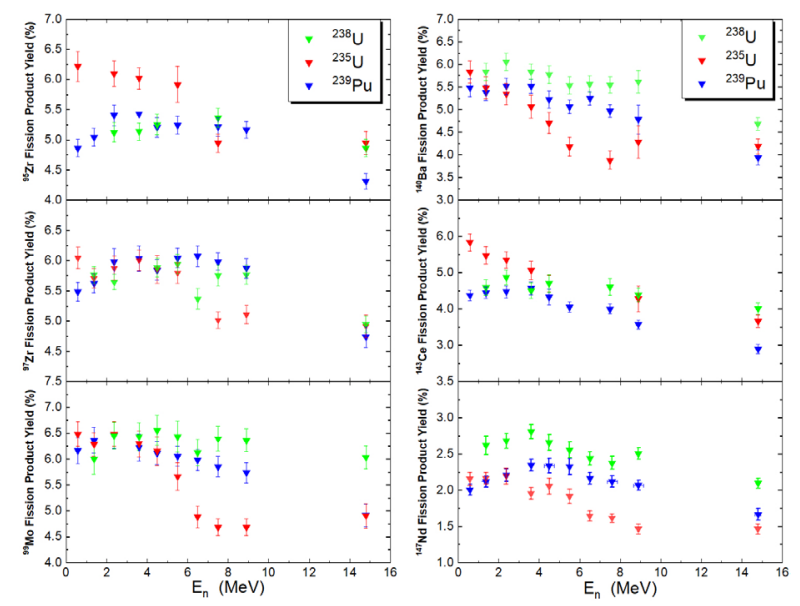

Figure 3. Fission product yield of ${ }^{95} \mathrm{Zr}$ (left-top), ${ }^{97} \mathrm{Zr}$ (leftcenter), ${ }^{99} \mathrm{Mo}$ (left-bottom), ${ }^{140} \mathrm{Ba}$ (right-top), ${ }^{143} \mathrm{Ce}$ (rightcenter), and ${ }^{147} \mathrm{Nd}$ (right-bottom) from the fission of ${ }^{235} \mathrm{U},{ }^{238} \mathrm{U}$, and ${ }^{238} \mathrm{Pu}$ in the $0.5 \mathrm{MeV}$ to $15 \mathrm{MeV}$ neutron energy range.

radiations were typically of a few days in duration, fission products with half-lives less than a few hours were not accessible to these studies due to saturation of their activities during the long irradiations.

\subsection{Measurement of short-lived fission-product yields}

As we approach the completion of our measurements of high-yield fission products with long half-lives (days, weeks or months referred to as long-lived chain fission products) [24], we have performed exploratory FPY measurements at $E_{n}=4.5,6.5 \mathrm{MeV}$ and $9.0 \mathrm{MeV}$ with a focus on isotopes with half-lives between approximately a few minutes and hours, referred to as short-lived fission products. One preliminary result from the short (1 hour) irradiations at TUNL are shown in Fig. 4 where we have measured more than 45 fission products spanning 30 unique mass chains for neutron-induced fission of ${ }^{235,238} \mathrm{U}$ and ${ }^{239} \mathrm{Pu}$ at $E_{n}=9.0 \mathrm{MeV}$. This is a substantial increase in data, particularly for the short-lived fission products, where almost twice as many masses have been measured compared to the long (days) irradiations. As can be seen from Figs. 1 and 4 , in addition to our already published fission chain yield of ${ }^{133} \mathrm{Xe}\left(t_{1 / 2}=5.2 \mathrm{~d}\right.$ ) [24] we added the FPYs for its precursors $-{ }^{133 m} \mathrm{Te}\left(\mathrm{t}_{1 / 2}=55.4 \mathrm{~m}\right),{ }^{133} \mathrm{Te}\left(\mathrm{t}_{1 / 2}=12.4 \mathrm{~m}\right)$ and ${ }^{133} \mathrm{I}\left(\mathrm{t}_{1 / 2}=20.8 \mathrm{~h}\right)$ in the isobaric mass chain $\mathrm{A}=133$ [30]. Note that this data also provides a time-dependent cumulative fission-product isomeric ratio of ${ }^{133 m} \mathrm{Te}$ and ${ }^{133 g} \mathrm{Te}$, an important observable for constraining the average angular momentum partitioned between the fission fragments. Measuring the very short-lived fission precursors of ${ }^{133} \mathrm{Te}$ will enable us to obtain the independent-yield ratio between a low-lying isomeric state and the nuclear ground state. Such data provides constraints on theoretical models $[31,32]$ regarding level densities, radiative strength functions and the completeness of the discrete level structures. These properties are frequently invoked in developing var- 
ious nuclear-data libraries that are used in a range of applications that require a sound knowledge of fission.

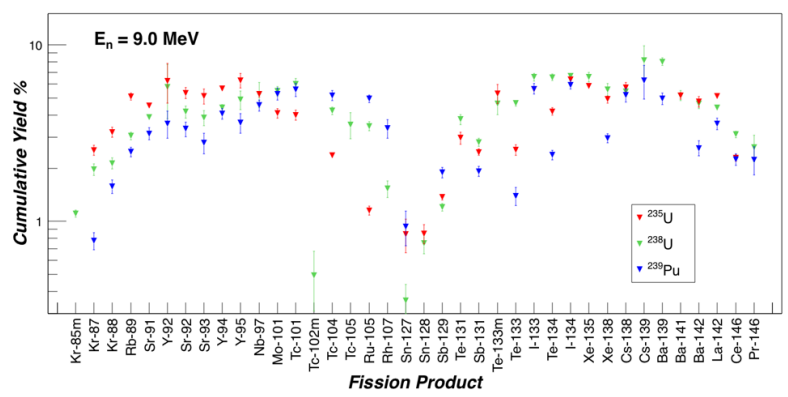

Figure 4. Preliminary short-lived FPYs from neutron induced fission of ${ }^{235} \mathrm{U},{ }^{238} \mathrm{U}$, and ${ }^{238} \mathrm{Pu}$ at $E_{n}=9.0 \mathrm{MeV}$. The irradiation time for all three fission targets was 1 hour.

\subsection{Measurement of very short-lived fission-product yields}

Since the most abundant independent yield fission products in every fission chain generally have half-lives in the range of seconds to minutes, it has become desirable to develop faster transport system for FPYs measurements. The new experiments will employ the same DFC used in the previous measurements to provide the most accurate determination of the total number of fissions during the irradiation [21]. The DFC will be located in close proximity $(4 \mathrm{~cm})$ to the neutron source. Performing a short irradiation allows us to access a new "region" of shortlived fission products (few seconds to minutes) which were oversaturated during our long irradiations and explicitly omitted from the previous analysis. The number of fission product isotopes accessible in specified half-life ranges is compared in Fig. 5. For example, during the long irradiation campaign, we measured 16 FPY, from 16 unique masses [24] out of 108 available from mass chain yield $80<A<160$. Adding FPY measurements of short-lived isotopes provides important information on the mass distribution, and also allows us to add data closer to symmetry and the wings in the mass distribution. The very shortlived FPY data will be extremely valuable for phenomenological fission codes like FREYA [38], CGMF [39], and GEF [40] to constrain the evolution of the mass distributions as a function of the incident neutron energy. In addition, the FPY data obtained in this work will utilize the recently released Fission Induced Electromagnetic Response (FIER) [41] code to ensure consistency across all irradiation time periods and experiments.

These new experiments utilizes a RApid Belt-Driven Irradiated Target Transfer System (RABITTS), which will move the fission target from close proximity to the neutron source, $24.5 \mathrm{~mm}$, to a low-background counting area. RABITTS is comprised of a linear actuator, controlled by a high power servomotor. Compared to traditional pneumatic systems, this approach has the advantages of an extremely high repeatability of $\pm 33 \mu \mathrm{m}$ for the irradiation and counting positions, consistent target alignment and

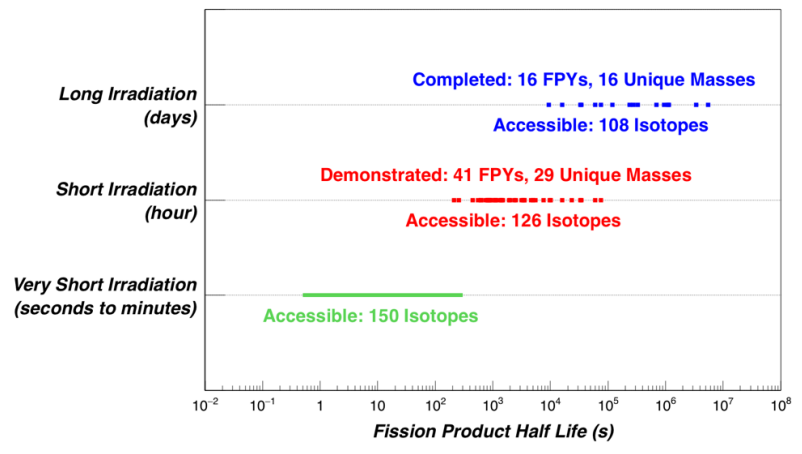

Figure 5. FPYs with half-lives greater than 1 hour measured by the LLNL-LANL-TUNL collaboration from long irradiation (blue), recently demonstrated from short irradiation (red), and very short (green) proposed to be explored in near future

orientation, and a controlled soft acceleration and deceleration. The system is fully automated, with user programmable irradiation time, counting time, and transit speeds up to $10 \mathrm{~m} / \mathrm{s}$. The system is designed to continuously cycle between irradiation and counting positions, performing cyclic activation [42], until the desired statistical accuracy has been reached.

Two RABITTS were implemented: a $1 \mathrm{~m}$ track with $0.40 \mathrm{~s}$ transfer time and a $7.19 \mathrm{~m}$ track with $1.09 \mathrm{~s}$ transfer time. The $1 \mathrm{~m}$ track was designed for measuring the shortest lived fission products and commissioned in December 2018. The longer, $7.19 \mathrm{~m}$ track, was commissioned in June 2019. This longer track moves the target through a narrow opening in a concrete shielding wall into a counting station located in a separate room. Despite the slightly longer transit time, the low-background environment present in this room still allows for high quality measurements of fission products with half-lives $<1 \mathrm{~s}$.

The low background counting area utilizes the exact same $\gamma$-ray spectroscopy techniques as our measurements of cumulative FPYs, consistently extending our measurements to include independent FPYs. High-Purity Germanium (HPGe) detectors are used to perform $\gamma$-ray spectroscopy and quantify fission products. These detectors are recorded in a list-mode digital data acquisition (DAQ) system, where events are time stamped with accuracy $<0.1 \mu \mathrm{s}$. A signal from RABITTS inputs into this same DAQ, fully synchronizing the $\gamma$-ray spectroscopy with the timing of the transfer system. Furthermore, a copy of the RABITTS signal is utilized to pulse the charged particle beam. The proton or deuteron beam is deflected during the transit and counting periods. This ensures that the target is not exposed to additional neutrons during the transit period, and prevents contamination by neutron-induced backgrounds in the HPGe detectors. The RABITTS control, DAQ, and beam pulsing are fully synchronized by design, ensuring the high fidelity of our time-dependent spectroscopy measurements.

The $1 \mathrm{~m}$ system was first benchmarked by measuring the isomer production cross-sections ${ }^{197} \mathrm{Au}\left(n, n^{\prime}\right){ }^{197 m} \mathrm{Au}$ and ${ }^{90} \mathrm{Zr}\left(n, n^{\prime}\right)^{90 m} \mathrm{Zr}$. A $\gamma$-ray spectra from the ${ }^{197 m} \mathrm{Au}$ experiment may be seen in Fig. 6. Although well shielded 
from the neutron beam, the HPGe detectors still see a large background during irradiation cycles. This background is greatly reduced during the counting cycles, when the neutron beam is not present. The decay curve for ${ }^{197 m} \mathrm{Au}$ is shown in Fig. 7. The half-lives of the isomeric states were measured as $t_{1 / 2}\left({ }^{197 m} \mathrm{Au}\right)=7.75 \pm 0.09 \mathrm{~s}$ and $t_{1 / 2}\left({ }^{90 m} \mathrm{Zr}\right)=$ $0.787 \pm 0.008 \mathrm{~s}$, in agreement with the NNDC values $[43,44]$.

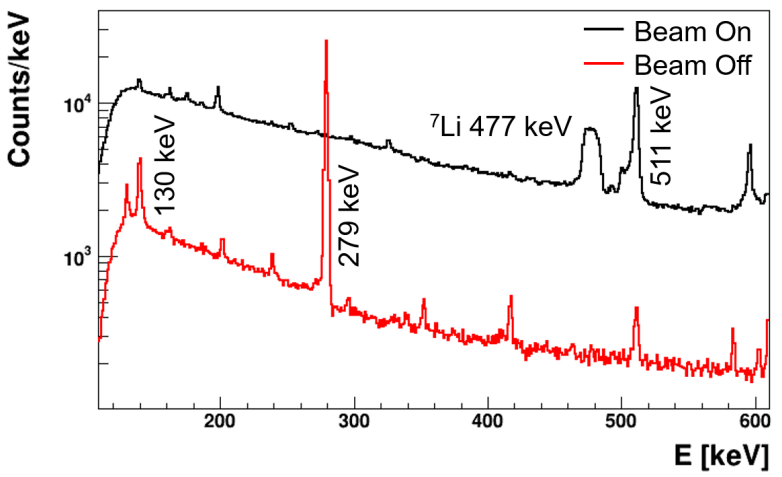

Figure 6. The $\gamma$-ray spectra during irradiation (black) and following irradiation (red) of ${ }^{197 m} \mathrm{Au}$ using the $1 \mathrm{~m}$ RABITTS. The reduction in $\gamma$-ray background due to the beam pulsing system may be clearly seen, along with the very intense 130 and 279 $\mathrm{keV}$ transitions from the decay of ${ }^{197 m} \mathrm{Au}$ [43].

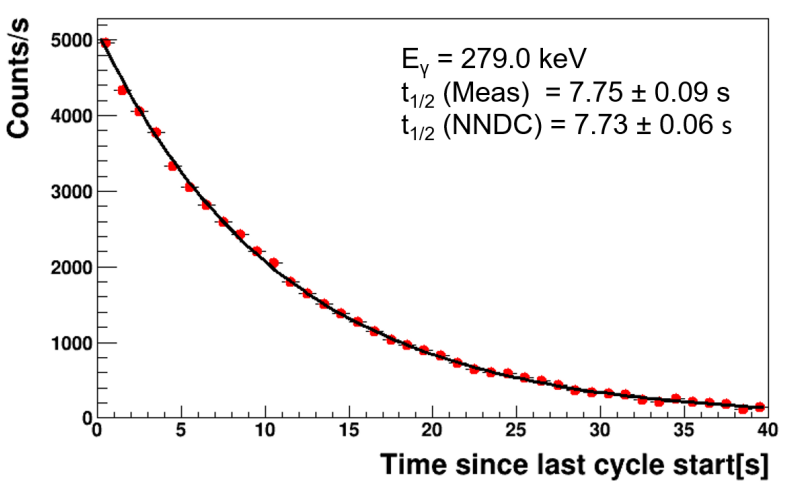

Figure 7. Measured decay data of the ${ }^{197 m} \mathrm{Au}$ isomer (red points), showing excellent agreement with the NNDC value (black curve) [43]. The ${ }^{197 m} \mathrm{Au}$ decay was measured using activation cycles of $40 \mathrm{~s}$ irradiation with $E_{n}=2.0 \mathrm{MeV}$ neutrons, followed by $40 \mathrm{~s}$ counting.

We have begun performing the same systematic study of the energy dependence of short-lived FPYs between $E_{n}=0.56$ and $14.8 \mathrm{MeV}$ at the same incident energies for which we now have data for the long-lived, high-yield fission products of ${ }^{235} \mathrm{U},{ }^{238} \mathrm{U}$ and ${ }^{239} \mathrm{Pu}$. Data has already been collected using RABITTS at $E_{n}=2.0$ and $4.5 \mathrm{MeV}$. Example $\gamma$-ray spectra are shown in Fig. 8. These spectra show the decay of ${ }^{96} \mathrm{Sr}\left(t_{1 / 2}=1.07 \mathrm{~s}\right)$ and ${ }^{91} \mathrm{Zr}\left(t_{1 / 2}=\right.$ $2.1 \mathrm{~s})$. This data represents the accumulation of 24 hours of total experimental time with repeating cycles of $3 \mathrm{~s}$ irradiation and $9 \mathrm{~s}$ counting, in a statistical uncertainty of
$2 \%$ for these two fission products. By measuring both the $\gamma$-ray energy and decay half-life with high resolution, we are able to unambiguously identify fission products without contamination from competing $\gamma$ rays. In addition to using the same $\gamma$-ray spectroscopy technique and the same fission chambers, the same thick $\left(200 \mathrm{mg} / \mathrm{cm}^{2}\right)$ targets are used in this experiment as the long-lived FPY experiments. Although our experimental infrastructure has been upgraded with the addition of RABITTS, the use of consistent techniques allows for consistent systematic uncertainties as we move from long-lived to very-short lived FPYs. The end result is a continuous set of FPY meaurements, with half-lives ranging from seconds to months, across incident neutron energies of $E_{n}=0.57$ to $14.8 \mathrm{MeV}$.
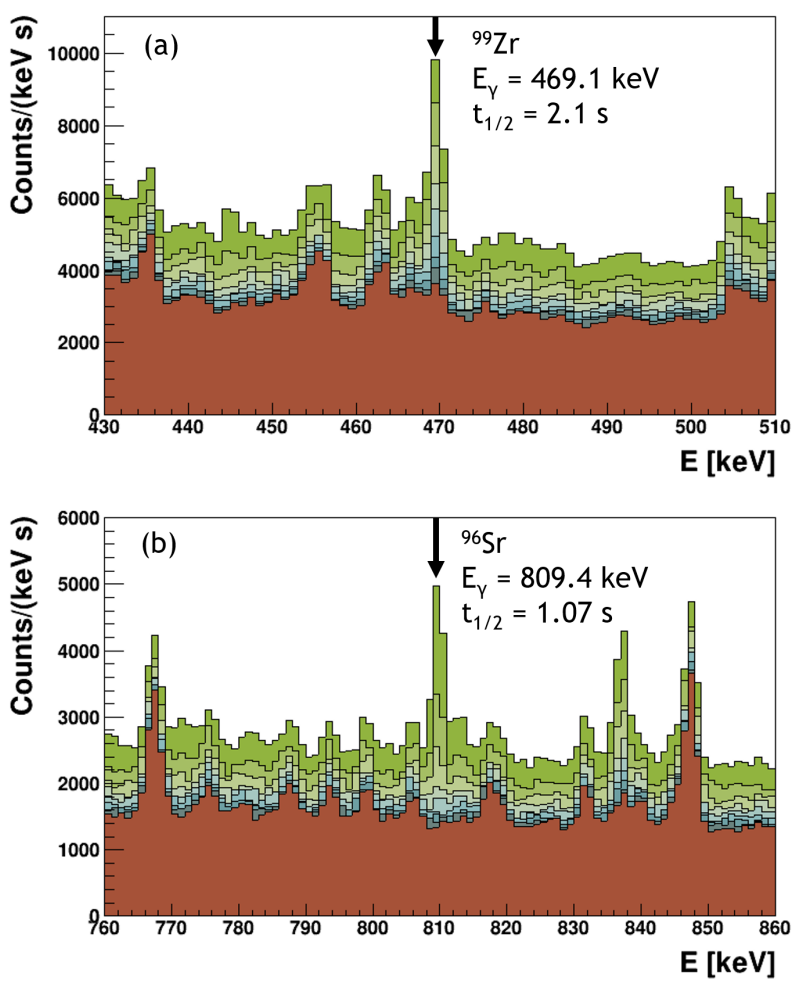

Figure 8. Gamma-ray spectra following the fission of ${ }^{238} \mathrm{U}$ by $E_{n}=2 \mathrm{MeV}$ neutrons in $1 \mathrm{~s}$ intervals. The data were collected using cyclic activation with $9 \mathrm{~s}$ counting time, following $3 \mathrm{~s}$ of irradiation. The strong transitions from ${ }^{99} \mathrm{Zr}$ (a) and ${ }^{96} \mathrm{Sr}$ (b) are both shown. The identity of these very-short lived fission products are confirmed by measurement of their $2.1 \mathrm{~s}$ (a) and $1.07 \mathrm{~s}$ (b) half-lives.

\section{Summary}

We have begun performing self-consistent FPY measurements for species with half-lives of seconds to minutes. The measurements will provide self-consistent, highprecision, and time-dependent FPY data for ${ }^{235} \mathrm{U},{ }^{238} \mathrm{U}$, and ${ }^{239} \mathrm{Pu}$ isotopes using monoenergetic and pulsed neutron beams for incident energies from 0.5 to $15.0 \mathrm{MeV}$. Using irradiations of varying duration, and a new automatic target-transfer system for short-lived fission products, we 
will measure the energy dependence of the cumulative and independent yields for more than two dozen fission products. The new measurements will give a complete picture of the fission product yield landscape; from the initial distribution produced directly by fission, through the complex, time-dependent evolution of the yields from $\beta$ decay and neutron emission, and finally the "end of the road" of the decay, the final distribution of stable nuclei. The uniqueness of these FPY data will provide insights crucial for the development of fission theory as well as important benchmarks for new data evaluations based on high-precision fission products.

This work was performed under the auspices of US DOE by LLNL under contract DE-AC52-07NA27344. We also want to acknowledge the supported by the US Department of Energy, Office of Nuclear Physics, under Grant No. DE-FG02-97ER41033, and by the National Nuclear Security Administration under the Stewardship Science Academic Alliance Program through the US Department of Energy, Grant No.DE-NA0003884.

\section{References}

[1] R. Wandenbosch and J. Huizenga, Nuclear Fission (Academic Press, Inc., New York, 1973).

[2] C. Wagemans, The Nuclear Fission Process (CRC Press, Inc., Boca Raton, Florida, 1991).

[3] F. Gönnenwein: C. Wagemans (Ed.), The Nuclear Fission Process, CRC Press, N. W., Boca Raton, Florida 33431, 1991, pp. 287-473.

[4] M. Lammer, Final report of a coordinated research project 1991-1996, IAEA-TECDOC-1168.

[5] H.O. Denschlag, Journal of Radioanalytical and Nuclear Chemistry, 203, 319 (1996).

[6] D.R. Nethaway, A. E. Richardson, Phys. Rev., 182, 1251 (1969).

[7] D.R. Nethaway, B. Mendoza, Phys. Rev. C, 6, 1827 (1972).

[8] D.R. Nethaway, G. W. Barton, Lawrence Livermore National Laboratory Report No. UCRL-51458 (1973).

[9] D.R. Nethaway et al., Phys. Rev. C, 16, 1907 (1977).

[10] L.E. Glendenin et al., Phys. Rev. C, 22, 152 (1980).

[11] L.E. Glendenin et al., Phys. Rev. C, 24, 2600 (1981).

[12] J.E. Gindler et al., Phys. Rev. C, 27, 2058 (1983).

[13] J.K. Dickens, Nuclear Science and Engineering, 70, 177 (1979).

[14] J.K. Dickens and J.W. McConnel, Nuclear Science and Engineering, 73, 42 (1980).

[15] J.K. Dickens et al., Nuclear Science and Engineering, 77, 146 (1981).
[16] J.K. Dickens and J.W. McConnel, Phys Rev. C 34, 722 (1986).

[17] J. Laurec et al., Nucl. Data Sheets, 111, 2965 (2010).

[18] J. Lestone, Nucl. Data Sheets 112, 3120 (2011).

[19] T. Kawano, M.B. Chadwick, J. Nucl. Sci. Technol. 50, 1034 (2013).

[20] T.R. England and B.F. Rider, ENDF-349, LA-UR94-3106 (1994).

[21] J.A. Grundl, D.M. Gilliam, N.D. Dudey and R.J. Popek, Nucl. Tech. 25, 237 (1975).

[22] C. Bhatia et al., Nucl. Instr. Meth. Phys. Res. A 757, 7 (2014).

[23] C. Bhatia et al., Phys. Rev. C 91, 064604, (2015).

[24] M. Gooden et al., Nucl. Data Sheets 131, 319 (2016).

[25] A.P. Tonchev et al., 6th Intern. Conf. on Fiss. and Prop. of Neutron-Rich Nuclei, p.381 (2017).

[26] M.B. Chadwick, T. Kawano, et al., Nuclear Data Sheets, 111, 2923 (2010).

[27] I. Thompson, et al., Nucl. Sci. Eng. 171, 85 (2012).

[28] J. Randrup and P. Moller, Phys. Rev. C 88, 064606 (2013).

[29] N. Schunck, D. Duke, and H. Carr. Phys. Rev. C, 91, 034327 (2015).

[30] J.A. Silano et al., submitted to Phys. Rev. C.

[31] I. Stetcu, P. Talou, T. Kawano, and M. Jandel, Phys. Rev. C 88, 044603 (2013).

[32] I. Stetcu, P. Talou, T. Kawano, and M. Jandel, Phys. Rev. C 90, 024617 (2014).

[33] S. Stave et al., J. Radioanal. Nucl. Chem. 307, 2221 (2016).

[34] P. Talou, R. Vogt et al., Eur. Phys. J A 54, 9 (2018).

[35] S. Okumura, T. Kawano, et al., J. Nucl. Sci. Technol. 55, 1009 (2018).

[36] A. C. Wahl, Rep. NEA/NSC/DOC (92)9 (OECD) (1992) 334-345.

[37] J.H. Choi et al., Phys. Rev. Lett. 116, 211801 (2016).

[38] J. M.Verbeke, J. Randrup and R. Vogt. Comp. Phys. Comm. 191, 178 (2015).

[39] P. Talou, T. Kawano and I. Stetcu, Nucl. Data Sheets, 118, 195 (2014).

[40] K-H Schmidt, B. Jurado, C. Amouroux and C. Schmitt, Nucl. Data Sheets, 131, 107 (2016).

[41] E.F. Matthews et al, Nuclear Inst. and Methods in Physics Research, A 891, 111 (2018).

[42] W.W. Givens, W.R. Mills, and R.L. Caldwell. Nucl. Instr. Meth. 80, 95 (1970).

[43] H. Xiaolong and Z. Chunmei, Nucl. Data Sheets, 104, 283 (2005).

[44] E. Browne, Nucl. Data Sheets, 82, 379 (1997). 